\title{
Design and Implementation of an Integrated Platform for Purchasing Optimization and Decision Making
}

\author{
Guoning Gao ${ }^{1, a}$, Jianrong Gong ${ }^{1, b}$, Junli Wu ${ }^{2, c}$, Xin Luo ${ }^{3, d}$, Bo Zhao ${ }^{3, e, *}$ \\ ${ }^{1}$ State Grid Zhejiang Zhejiang Electric Power Trade Center, Hangzhou, Zhejiang, China \\ ${ }^{2}$ State Grid Zhejiang Economic Research Institute, Hangzhou, Zhejiang, China \\ ${ }^{3}$ Beijing Tsingsoft Technology Co., Ltd., Beijing, China
}

Keywords: Purchasing Planning, Load Forecasting Analysis, Units Planned Outage

\begin{abstract}
With the development of electric power industry, the government has not adapted the demand of electricity market reform. This platform presents a user friendly, powerful purchase plan editor to adapt to the current electricity market reform, standardize the purchasing of electricity planning business processes. According to the design, construction and deployment of the platform, the paper introduces the design ideas and construction results. Through the research of the advanced power generation planning method, the research results will be embedded in the system, which can enhance the scientific decision-making, and it is expected to realize the maximization of the interests of the power grid and power plant. And the platform of practical design concept, can greatly improve the working efficiency of the relevant staff.
\end{abstract}

\section{Introduction}

In recent hundred years, the traditional power industry applied integrated management, including production, transmission, distribution and selling [1]. For the purpose of optimizing resource allocation, realizing profit transfer and encouraging power plants' energy-saving, variety of power markets globally had begun the power market restructions since late 1980s[2-4]. China started its power market-oriented reform since 1998, and in 2002 the State Council issued a document that put forward China's power market-oriented reform plan. In 2015, the State Council issued another document and its supportive documents, further promoting the market-oriented reform.

The trading plan of Grid Company mainly includes two aspects, the electricity contract decomposition and generation purchasing plan. The electricity contract decomposition is what trading center of Grid Company mainly concerns. Literatures [6]-[10] introduced different methods for contract decomposition, which were useable in some scenarios. Generation and purchasing plan has been studied and practiced across the border, and the optimization objectives includes purchasing cost, power grid security, energy-saving and transmission losses. Generation planning can been divided into short-term planning and long-term planning. Literatures [11]-[13] put forward three different day-ahead generation planning models. Literatures [14] and [15] put forward two different monthly generation planning models. Literature [16] put forward a multi-objectives 
grouping generation planning models based on the weights of professional's opinions, which is subjective but flexibility, and has been applied in many provinces. Literature [17] quantitatively evaluated the coordinate cost between economy and safety by solving multi-objectives optimization problem. The majority of foreign researchers focused on studying security-constrained economy dispatch and security-constrained unit commitment.

Regional Grid Company in China applied a traditional method to make trading plan in practice. At the beginning of the year, Grid Company would draw up yearly generation plan for every power plant and electricity purchasing price for every generating plant unit in its region according to the document issued by National development and Reform Commission. At the middle and end of the year, Grid Company would modify its previous yearly generation plan with other government departments, according to demand variation and power plants' generation status. The trading center of Grid Company would forecast monthly electricity supply in its region and develop a regional power line purchasing plan. When preliminary trading plan completed, trading center of Grid Company need to keep contact with dispatch department and power plants to ensure the feasibility of the plan. The current method is barely usable under market-oriented reform for a variety of reasons, including its low degree of informatization degree, low working efficiency and planning based on experience.

In this paper, complex constraints of power grid and features of power plants are considered and a new power purchasing optimization method based on reference schedule is applied. Meanwhile, the managements by objectives and processing are combined, and four evaluation indicators of trading plan's efficiency are designed, and a planned outage model is studied to satisfy the need of long-term planned outage. Based on the simulation and purchasing plan optimization method, a purchasing planning and analysis platform is built.

\section{A New Purchasing Optimization Method}

\subsection{A Purchasing Model Considering Grid's Complex Constraints}

In this paper a purchasing model considering grid's complex constraints is applied. This model uses monthly generating electricity of each power plant in each month as the independent variable to realize yearly and monthly trading plan optimization and multi-objectives coordinate optimization, while considering every constraint in power market.

\subsubsection{Independent Variable and Optimization Objective}

The independent variables of purchasing plan is generation plan and power line purchasing plan of every plant in every month. When Grid Company is in the process of drawing up purchasing plan, optimization objectives, including economy, energy, balance of different plants' generation plan and yearly project completion, are considered, and the specific calculations are in literature [20]. For trading center, economy and energy optimization objectives are definite numbers. Trading center mostly modify the difference of generating electricity of different units in one plant and coordinate surplus generating electricity in the allowed yearly generation plan deviation. The balance of different plants' generation plan and yearly project completion optimization objectives are key points for trading center, while the balance of yearly completion is priority.

\subsubsection{Constraints}

There are five constraints needing considerations. 1) System power balance constraints, which ensures the electricity balance in the trading plan. 2) Network constraints, which ensures essential buses and branches safe. 3) Coal storage constraints, which ensures plant coal usage is less than 
plant coal storage. 4) Generation limit, the plant generation would be limited because of capacity, reserve and loss of load probability. 5) Plan balance limit, when planning generation, all kinds of plants should fulfil their plan balancedly.

\subsubsection{Multi-Objectives Decomposition-Coordination Optimization}

\subsubsection{Decomposition-Coordination Optimization}

The optimization objectives, economy, energy saving and generation plan balance, are decomposed in purchasing optimization model considering complex constraints during different stages. Specifically, economy and energy saving optimization objectives are considered when planning plant yearly generation and power line purchasing, while the generation plan balance is considered when plan plants' monthly generation plans.

\subsubsection{Yearly Generation Plan Optimization and Correction}

When optimizing trading plan, yearly generation plan should be preset. For plant whose generating capacity is not enough to fulfil its generation plan, its balance charge could be replaced by other plants according to economy and energy optimization objectives. The difference of total generation plan and total decomposition plan could be balance according to a certain coordination principle.

\subsubsection{Monthly Generation Plan Rolling Optimization}

The generation plan rolling optimization is a dynamic generation plan method. Firstly, the generation plan in a certain period is optimized, then the future generation plan is rolling modified because of the implementation of plans. The rolling optimization plan method combines short-term plan and mid-term plan.

\subsection{A New Trading Plan Model based on Reference Schedule}

Section 2.1 introduces a model that treat schedule variance indifferently, which may lead large plant's schedule variance bigger to release space for small plants to optimize. Meanwhile, the mean value of all plants' generation should be calculated, which is hard for a large scale planning problem. Besides, the calculation basis is the mean value of all plants' schedule variance, which ignores individual difference between plants, e.g. plants' maintenance. To make up for the deficiency, this paper applied four reference schedule setting methods and two schedule variance weighting methods. A unified optimization model considering plant's reference schedule is,

$$
\min \sum_{m=T_{m}}^{T_{M}} \sum_{i=1}^{N_{P}} W_{P_{i}}^{m}\left|\rho_{P_{i}}^{m}-\bar{\rho}_{P_{i}}^{m}\right|
$$

$W_{P_{m}}^{m}$ is the schedule variance of plant $i$ in month $m$.

$\bar{\rho}_{P_{i}}^{m}$ is the reference schedule of plant $i$ in month $m$.

This model considers plant maintenance, power demand and preferences of decision makers, and is compatible with purchasing model based on grid complex constraints mentioned in section 2.1.

\section{Executive performance evaluation indicators}

There are four evaluation indicators from the angles of objectives managements and process managements in the process of execution. 


\subsection{The Correlation Coefficient of Average Load Rate}

By calculating each plant's load rate and network average load rate in the trading plan, the execution risk of trading plan is evaluated quantitatively.

\subsection{Monthly Average Reference Schedule Variance}

By applying the method of calculating the mean value of differences in all plants' planned schedule and reference schedule, considering the influence of execution risk because of reference schedule, a new indicator named monthly average reference schedule variance is defined.

\subsection{Yearly Generation Plan Completion Deviation}

All plants' yearly generation plan completion standard deviation is used as the quantitative evaluation indicator. The smaller the standard deviation is, the better the fairness of trading plan is.

\subsection{The Number of Operating on the Boundary Plants}

Plant's monthly load rate influences the dispatching department and plant itself. If plant is running on constraints' boundaries, which is so called operating on the boundary, the feasibility of dispatch and fairness of plant would be influenced, and brings risks to the execution of trading plan. By counting the number of operating on the boundary plants in the trading center, analyzing the reason of operating on the boundary, the actual risks in the trading plan is reflected, and it can provide reference for scheduling.

\section{Planned Outage Model based on Gradually Decreasing Reserve Method}

The generator planned outage is an important part in generators' dispatch, and is of the essence when designing an integrated platform.

\subsection{Current Generator Planned Outage Model}

One regional grid company uses following model to plan monthly generator outage.

1) Calculation daily equilibrium margin,

$$
\text { daily equilibrium margin =maximum output-maximum load-upper spinning reserve }
$$

2) According to the plant's monthly generation plan, maintenance plan and the principle that load rate should between $60 \%$ and $80 \%$, the planned outage is calculated.

The problem of this model is: 1) Only upper spinning reserve is considered, and lower spinning reserve and peak shaving do not take into consideration; 2) A new outage plan would be highly influenced by the old one; 3) The modifying process is complex, which makes it hard to consider all constraints. To overcome the weakness, this paper puts forward a new planned outage model, which uses an optimization method instead of old model to draw up a better outage plan.

\subsection{The New Planned Outage Model}

The new planned outage model put forward in this paper, which is based on gradually decreasing reserve method, is a long-term planned outage model. This model chooses to have as many as possible small capacity plants shut down in lowest load periods, and the generation capacity can be 
used as load, to ensure total load smoothly variant, also keeps total capacity smoothly variant.

Basic planned outage model contains four procedures,

1) Arrange the scheduled generating units by size, with the largest first and the smallest last.

2) Schedule the lowest generating unit for outage during lowest load periods.

3) Repeat step 2 by scheduling the next generation unit on the outage list until all the designated generating are scheduled.

Calculating planned outage model is the first step to plan units dispatch. The advantages of this model are,

- Model considers daily capacity reserve, spinning reserve, which ensures the safety and stability of power system.

- The modifying process of this method is relatively easy, and it can draw up long-term outage plan and rolling outage plan.

- This planned outage model is not influenced by original plan, which ensures to find global optimum.

\subsection{Planned Outage Model Constraints}

Basic planned outage model considers electricity balance problem in power system and ignores other constraints, which is impractical. To fulfil this problems, unit monthly maximum start-up times, unit minimum run time, minimum off time and units start-up fee.

Unit monthly maximum start-up times ensures plant load rate. Unit minimum run time and minimum off time ensure the safety of generating unit. Unit start-up fee ensures the cost of outage.

\section{Software Platform Designing}

\subsection{Function Module Content}

The whole system contains four function modules, which are basic data management, load forecasting, power balance analysis and purchasing plan. The platform has a standard system interface and information query functions.

\subsection{Process Implementation}

The integration of power purchase optimization, considering load forecasting and purchasing marginal conditions, is based on plants' reference schedule.

\subsubsection{Basic data management}

With the using of needed data supplied by basic data management in the means of table and graph, it is more convenient for decision maker to verify and decide the optimization objectives.

\subsubsection{Load forecasting analysis}

This function is based on daily load curve simulation forecasting method, which deeply analyzes the correlation between electrical power and electrical power indicators, from the points of electricity and electrical power. This forecasting method simulate daily load curve for the forecasting month, and characteristic values are accumulated to overcome the disadvantages of traditional monthly electricity forecasting method. 


\subsubsection{Power electricity balance}

The main function of electricity balance is to assist electricity forecast, power line purchasing plan, wind electricity and power plants' generation plan. Power balance analysis function module calculates daily equilibrium margin using power balance and monthly power balance came from daily analysis, considering purchasing plan. If power is not balance, it is hard to plan monthly generation, and the boundary conditions of purchasing plan and generation plan are needed to be modified. All boundary conditions needed to take into consideration are unit maintenance, planned outage, block, power line purchasing plan, wind electricity and load. The function module also support monthly power balance analysis.

\subsubsection{Purchasing plan precompiling}

At first, yearly maintenance plan of all power plants are analyzed to get monthly adjustable capacity and maximum generating output. Then, based on the results above, plant units whose yearly generation plans are unreasonable are separated, according to yearly generation plans of all plant units. Finally, the reference schedule of generation plan is set. For every plant unit, the deviation of actual generation plan is quantitatively analyzed, and those plans whose reference schedule and load rate are not matched would be paid more attention.

\subsubsection{Purchasing plan compiling}

The decision makers could accept the system default boundary conditions, or change the boundary conditions below by themselves, global constraints on all plants, individual constraints on plants who are against global constraints, and some plants' load rate or generation electricity are set, while some types plant's load rate range are set. There are multiple choices for optimization objectives and models, including minimizing plant load rate deviation, cumulative progress deviation and cumulative electricity deviation.

By saving the setting, different cases could be compared and analyzed, and every auxiliary indicators could be showed by visualization to assist traders draw up final trading plan.

\subsubsection{Units planned outage}

Units planned outage modified based on given trading plan, considering all kinds of constraints, and builds a unit commitment model to give the outage plan. For those trading plan which do not have feasible solution, an advice would be given. Outage plan has no limit on number of days, which means model can be used to do monthly plan or weekly plan. Applying planned outage trading plan adjustment method, grid operation constraints are taking into consideration. Based on exist trading plan, this function module can plan unit monthly outage to verify if trading plan works. Besides this function module can adjust plants' generation in batches or individual.

\subsubsection{Generation plan releasing}

The system support store but not release an adjusted plan. By comparing multi stored cases, a final trading plan can be assured and released.

\section{The Calculation Example and Platform Introduction}

\subsection{The Calculation Example}

This section shows the main functions interface of the platform. Figure 1-4 show load 
forecasting interface, power balance interface, unit planned outage interface and trading plan interface.

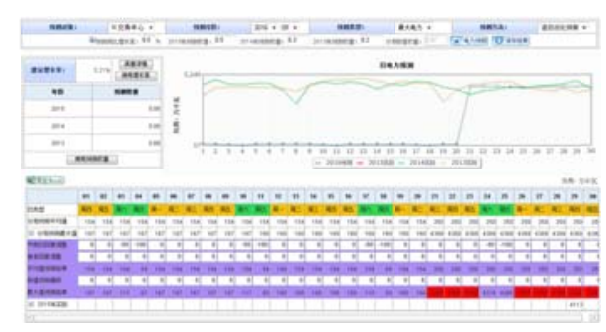

Figure 1 Load Forecasting Interface

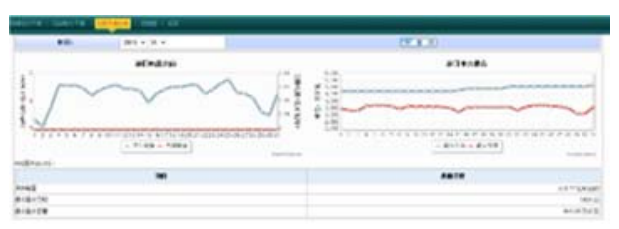

Figure 2 Power Balance Interface

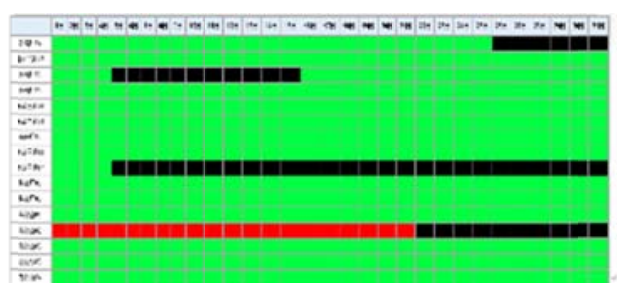

Figure 3 Unit Planned Outage Interface

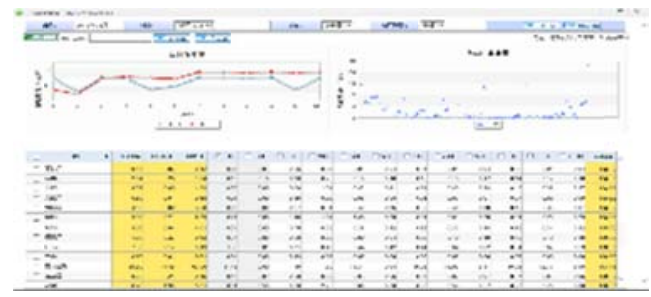

Figure 4 Trading Plan Interface

\subsection{Platform Introduction}

This platform present a user-friendly, functional purchasing plan editor, because of intensive layout, configuration and presentation. Operators could choose all kinds of auxiliary information display' visibility and orders, and can easily sort and screen needed data. For those whose operators need pay attention to, this platform would change or highlight its color. This function module provide a flexible tool for generation plan modify.

\section{Conclusion}

Based on the actual needs in the construction of power market, this paper applies considering complex constraints and reference schedule purchasing plan model, and combines performance evaluation indicators to warn trading risk, planned outage model to develop an outage plan. Based on the research above, integrated platform is designed and implemented. Using the actual data supported by a power trading center to demonstrate and evaluate the effectiveness and rationality of 
purchasing model and software platform.

\section{References}

[1] Songhuai Du. Power Market (Edition Three) [M]. Beijing: China Electric Power Press, 2008.

[2] Fan li, Min Song, Yonghua Song. Review and Prospect of Restructuring Electricity Market Modes in United Kingdom [J]. International Electric Power for China, 1999(1):50-54.

[3] Lianjun Shi, Fang Han. Nordic Power Market and Its Operation [J]. International Electric Power for China, 2000(2):4-9.

[4] Bin Wang, Jianjian Jiang, Chongqing Kang, etc. Main Concepts of Standard Market Design in United States and its Enlightenments to Electricity Market Design in China [J]. Power System Technology, 2004,28(16):21-26.

[5] Canbin Li, Chongqing Kang, Qing Xia, etc. Study on Trading Mechanism of Regional Electricity Market [J]. Power System Technology, 2004,28(7):34-39.

[6] Tiechao Dai, Dan Zhang. Application of Resoluting Computation of Decisive Contract Electric Energy in Zhejiang Generation Power Market [J]. East China Electric Power, 2000(10):7-9.

[7] Jianhua Chen, Ning Zhang, Tiechao Dai, etc. Empirical Analysis on the Deterministic Contract Decomposition Algorithm [J]. Automation of Electric Power Systems, 2007,31(11):26-30.

[8] Canbin Li, Yajie Hu, Hongjun Zhao, etc. General Model and Algorithm for Contract Energy Decomposition [J]. Automation of Electric Power Systems, 2007,31(11):26-30.

[9] Shuai Wang. A Study of Rolling Generation Schedule Optimization Method [D]. Beijing: North China Electric Power University, 2009.

[10] Pengfei Su. Study on the Electric Contract Decomposition Strategies Considering Uncertain Factors [D]. Shandong: Shandong University, 2010.

[11] Yu Shen, Qing Xia, Jianjian Jiang, etc. a Novel Model of Short-term Trade Planning in a deregulated Power System [J]. Automation for Electric Power Systems, 1999,23(18):12-16.

[12] Lingyan Wang, Zili Zhang, Juan Shu, etc. Coordination of the Dispatching Relationship Between the Contract Volumn and Competitive Bidding Volumn in Daily Generation Scheduling [J]. Automation of Electric Power Systems, 2008,32(17):26-29.

[13] Zhifei Liang, Qing Xia. An Accurate Daily Generation Scheduling Model and Application Algorithm [J]. Automation of Electric Power Systems, 2008,32(17):26-29.

[14] Yi Mao, Wenyan Che. Monthly Generation Scheduling Considering Energy-saving and Economic Benefit [J]. Modern Electric Power, 2008,25(5):73-78.

[15] Zhifei Liang, Qing Xia, Hongqing Xu, etc. Monthly Generation Scheduling Method Based on Muti-Objectiveive Optimization Model for Provincial Power Grid [J]. Power System Technology, 2009,33(13):90-95.

[16] Liangyuan Wang, Wenbin Liu, Risheng Fang, etc. A Multi-Objectiveive Clustering-Based Method to Draft Medium- and Long-Term Schedules for Power Generation and Purchase and Its Application [J]. Power System Technology, 2011,35(6):167-170.

[17] Xin Sun, Yuechun Lv, Jun Gao, etc. Power Grid Economy and Security Coordination Lean Method [J]. Power System Technology, 2009(11):12-17.

[18] Mitani T, Mishima Y, Satoh T, et al. Optimal Generation Scheduling under Competitive Environment: IEEE International Conference on Systems, Man, and Cybernetics, Taipei, Taiwan, 2006 [C].

[19] Jabr R A, Coonick A H, Cory B J. A study of the homogeneous algorithm for dynamic economic dispatch with network constraints and transmission losses [J]. IEEE Transactions on Power Systems, 2000,15(2):605-611.

[20] Hui Liu. Study on the Strategy of Economic Dispatch in Power Generation Scheduling of Beijing Power Grid 
[D]. Beijing: North China Electric Power University, 2013.

[21] Yuguo Chen, Yi Wang, Jialong Li, Sijie Liu. Bilateral Energy Trade Contract Decomposition Model and Results Evaluation [J]. Southern Power System Technology, 2015(8):32-37. 